\title{
MODELOS MATEMÁTICOS PARA ESTIMATIVA DE EXPORTAÇÃO DE NUTRIENTES EM POVOAMENTOS DE Pinus taeda L.
}

\author{
Luiz Moro*, Carlos Bruno Reissmann**, Helton Damin da Silva***, Carlos Alberto Ferreira**** \\ Osmir José Lavoranti *** \\ *Eng. Florestal, Dr., Valor Florestal - luiz.moro@valorflorestal.com.br \\ **Eng. Florestal, Dr., Depto. de Solos e Engenharia Agrícola, UFPR - reissman@agrarias.ufpr.br \\ ***Eng. Florestal, Dr., Embrapa Florestas - helton@cnpf.embrapa.br - osmir@cnpf.embrapa.br \\ ****Eng. Agrônomo, Dr., Embrapa Florestas - calberto@cnpf.embrapa.br \\ Recebido para publicação: 03/07/2006 - Aceito para publicação: 30/09/2006
}

\begin{abstract}
Resumo
Este trabalho teve como objetivo desenvolver e avaliar a aplicabilidade de modelos matemáticos para quantificar a exportação de nutrientes, na colheita de floresta de Pinus sp. O estudo ocorreu em plantios de $P$. taeda em 4 idades, localizados na região de Guarapuava (PR). As amostras foram retiradas na base, $1,0 \mathrm{~m}, 1,3 \mathrm{~m}, 2,0 \mathrm{~m}$ de altura e deste ponto em diante a cada metro até altura comercial. Destas amostras foram determinados os teores dos macronutrientes, do lenho e casca, no laboratório da EMBRAPA - Florestas - Colombo (PR). A amostragem aleatória em povoamentos de $P$. taeda em diferentes idades, nos estágios vegetativos: dominante, co-dominante e dominada e em diferentes condições de manejo e produtividade, permite obter equações de estimativas de acúmulo nutrientes com altos índices de correlações e confiabilidade. A obtenção de amostragem em segmentos até 4,0 $\mathrm{m}$ de altura possibilita determinar o conteúdo total dos nutrientes, sem necessidade de amostragens nos segmentos de posição mais elevada da árvore. A determinação do nutriente nitrogênio apresenta um maior grau de complexidade e requer basicamente que outros macronutrientes também sejam determinados, o que demanda maiores custos amostrais e laboratoriais.
\end{abstract}

Palavras-chave: Pinus taeda; sustentabilidade; exportação; nutrientes; modelos matemáticos.

\begin{abstract}
Mathematic models for nutrient exportation estimate of Pinus taeda L. stands. This study aims at developing and assessing the usage of mathematical patterns to quantify the nutrient exportation in harvest of Pinus forests. This study to be carried out, in plantations of Pinus taeda with 4 ages, located in Guarapuava (PR) region. The samples were taken from the base, $1.0 \mathrm{~m}, 1.3 \mathrm{~m}, 2.0 \mathrm{~m}$ high, and from this point on, at each meter until it reached commercial height. Through these samples it was determinate the content of macronutrients, from the wood and the bark, being carried out at the Embrapa Floresta laboratory, in Colombo (PR). The random samples taken from Pinus taeda populations at different ages, in vegetative strata: dominant, co-dominant and suppressed), different management conditions and yield levels, allow to obtain estimation equations of nutrient accumulation with high levels of correlations and reliability. Obtaining samples in segments up to $4 \mathrm{~m}$ high allows determine the total amount of nutrients, without the need to have additional samples from segments of higher position in relation to the tree top. The determination of nitrogen content shows higher complexity and, basically, requires that others macronutrients be also determined, which implies in higher sampling and laboratory costs.

Keywords: Pinus taeda; sustainability; exportation; nutrients; mathematical patterns.
\end{abstract}

\section{INTRODUÇÃO}

O Pinus taeda tem sua ocorrência natural do sul dos Estados Unidos, ao leste do estado do Texas, distribuindo-se pelo vale do Rio Mississipi ao extremo sudeste dos estados de Oklahoma, Arkansas central e sul do estado do Tennessee (USDA FOREST SERVICE, 2000). Hoje os plantios de Pinus sp no Brasil ocupam extensas áreas que compõem aproximadamente 1.800 .000 hectares. Na região Sul, estima-se em 1.060.000 ha as áreas plantadas com Pinus sp., que muito vem contribuindo com o 
desenvolvimento da economia brasileira, constituindo a base para a produção de celulose e papel, embalagens, aglomerados, compensados e chapas, entre outras atividades industriais (SBS, 1998).

O desenvolvimento de mercado, a estabilidade da economia e a implantação de um parque industrial fizeram com que o consumo da madeira de Pinus sp. nos últimos anos crescesse de forma até preocupante, uma vez que a implantação das florestas não ocorreu na mesma ordem da colheita. Esse fato está ocasionando e poderá agravar ainda mais o descompasso em abastecimento de madeira oriunda dessa espécie, pressionando os preços para patamares mais elevados. Mantendo-se a demanda atual, o déficit da madeira de Pinus sp será acentuado entre 2007 e 2016, alcançando um pico máximo por volta de 2011. Nessa época, o déficit estimado é de 12 milhões de metros cúbicos com casca (VALOR FLORESTAL, 2004).

$\mathrm{Na}$ exportação de biomassa, é comum o uso de modelos matemáticos para estimar o volume individual do tronco, principalmente em florestas homogêneas plantadas. Os modelos também têm sido desenvolvidos para a estimativa dos pesos do tronco com e sem casca, da casca, galhos e folhas (REIS, 1985).

Daniels et al. (1979), em estudos desenvolvidos em Pinus taeda, efetuaram análise comparativa entre modelo em nível de povoamento, modelo por classe diamétrica e modelo para árvores individuais, e mostraram que os três tipos de modelos geraram estimativas acuradas de produção, indicando que a escolha do modelo de produção depende basicamente do nível de detalhamento que se quer.

Burkhart (1987) afirma que são muitos os métodos disponíveis para o ajuste simultâneo dos parâmetros das equações de um sistema e considera que devem ser observados vários pontos. Primeiramente, a finalidade de um sistema de crescimento e produção é efetuar estimativas, e poucos trabalhos foram feitos para ilustrar o desempenho dos sistemas cujo ajuste dos parâmetros é simultâneo. Uma segunda consideração é que estimativa simultânea envolve um compromisso, em que nenhum dos componentes do sistema seja ajustado pior do que se fosse estimado individualmente. Outro fato que deve ser considerado é que muitas das técnicas econométricas foram desenvolvidas para determinada hipótese de trabalho, e elas podem não ser apropriadas, em sua plenitude, a sistemas biológicos cujo objetivo é a predição.

Scolforo (1976b) desenvolveu um simulador de crescimento e produção, denominado PISAPRO, para povoamentos de Pinus taeda e P. elliottii de toda a área pertencente à PISA Florestal S.A. A esse simulador, foram associados critérios de análises de investimentos de alternativas de manejo, pelo uso do programa denominado INVEST, que permite a obtenção dos seguintes resultados:

a) estimativa presente do volume total, sortimentos e demais características do povoamento florestal por classe diamétrica, sítio, idade, espécie e região;

b) prognose do volume total, área basal, sortimentos, incremento médio anual e outras características do povoamento por classe diamétrica, sítio, idade, espécie e região; e,

c) simulações de desbastes pré-comerciais; sistemático na sexta linha combinado com seletivo; sistemático na décima linha combinado com seletivo; seletivo, para diferentes idades, intensidade de remoção e número de desbaste, até no máximo o quarto e corte final.

Scolforo e Machado (1988) apresentam discussão pormenorizada de como selecionar equações de maneira adequada, enfatizando que a escolha dos modelos simplesmente pelo $\mathrm{R}^{2}$ e pelo erro da estimativa (EE) pode levar a erros e que, portanto, a análise visual dos resíduos expressos graficamente é fundamental.

Silva (1996), estudando diversos modelos para estimativa de conteúdos de macronutrientes na casca de Eucalyptus grandis, nas idades de 3, 5 e 7 anos, obteve as melhores estimativas, com exceção apenas para o fósforo aos três anos, com as amostragens de casca nos seguintes segmentos de altura do tronco: para o nitrogênio e magnésio 1,0-1,3 $\mathrm{m}$, fósforo 1,3-2,0 $\mathrm{m}$, potássio e cálcio 1,3-2,0 $\mathrm{m}$.

Valeri (1988) não encontrou relações de dependências entre os teores de nutrientes dos componentes das árvores e as classes do diâmetro à altura do peito (DAP). Van Lear et al. (1984) também observaram que não existe relação próxima entre os teores de nutrientes e o peso de matéria seca de um componente. Andrae e Krapfenbauer (1983) não encontraram relações significativas dos teores de Mn de qualquer um dos componentes das árvores com as variáveis DAP, $\mathrm{H}$ e suas combinações. Para os ramos vivos, constataram que as concentrações de $\mathrm{N}, \mathrm{P}, \mathrm{K}, \mathrm{Ca}$ e $\mathrm{Mg}$ diminuíram com o aumento da classe de DAP e que o aumento da fração de madeira no total do material acarreta uma diminuição dos teores de nutrientes. 
Objetivou-se com o presente trabalho desenvolver modelos matemáticos que permitam a estimativa do acúmulo e exportação de nutrientes no lenho e na casca do Pinus sp nos diferentes desbastes e colheita final da floresta.

\section{MATERIAL E MÉTODOS}

\section{Caracterização da área de estudo}

A realização do presente estudo exigiu que fossem selecionados plantios de P. taeda com 8,12 , 18 e 23 anos de idade, localizados em povoamentos de $1^{\circ}, 2^{\circ}$ e $3^{\circ}$ desbastes e com corte raso da floresta, respectivamente. Os materiais nas idades de 8, 18 e 23 anos foram coletados, respectivamente, nos municípios de Guarapuava, Campina do Simão e Cantagalo, no estado do Paraná, provenientes da Manasa-Madeireira Nacional S.A. O material da idade de 12 anos foi obtido na empresa Santa Maria Papel e Celulose, no município de Guarapuava (PR). As áreas localizam-se aproximadamente na latitude

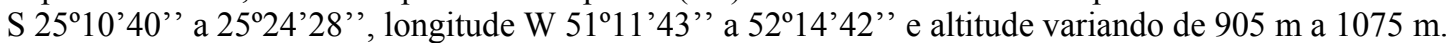

O clima da região de influência das empresas Manasa-Madeireira Nacional S.A. e Santa Maria Papel e Celulose é classificado, segundo Köppen, como sendo $\mathrm{Cfb}$, com verões frescos de temperatura média do mês mais quente inferior a $22{ }^{\circ} \mathrm{C}$ e do mês mais frio igual a $12{ }^{\circ} \mathrm{C}$, sendo durante onze meses maior que $10^{\circ} \mathrm{C}$ e com até cinco geadas por ano. A temperatura média anual é de $16,7^{\circ} \mathrm{C}$. A precipitação pluviométrica distribui-se regularmente durante o ano, variando entre 101,6 a 187,5 mm por mês, com média anual de $1.674,8 \mathrm{~mm}$. (SIMEPAR) ${ }^{1}$.

Segundo a Carta de Levantamento de Reconhecimento dos Solos do Estado do Paraná (EMBRAPA, 1984), ocorrem na região de Guarapuava, predominantemente, os seguintes solos: Latossolo Bruno Álico e Associação de Latossolo Bruno Álico e Cambissolo Álico; em menores quantidades, Cambissolo Húmico Gleico, Glei pouco Húmico, Solos Orgânicos e Solos Litólicos. Os solos predominantes das áreas de estudo são das classes de Latossolos Brunos e Cambissolos.

\section{Metodologia}

As amostras coletadas em campo constituíram-se de discos do tronco contendo lenho e casca, de 2 árvores para cada estrato (dominante, co-dominante e dominada) da floresta e nas quatro idades definidas, totalizando 24 árvores. Os materiais foram coletados entre o período compreendido de maio de 2002 a fevereiro de 2003. Para a amostragem do tronco, coletaram-se discos com lenho e casca a cada metro, a partir da base, incluindo o diâmetro à altura do peito (DAP), até à altura comercial, conforme metodologia utilizada por Silva (1996). Os discos foram imediatamente identificados com lápis hidrocor, caracterizando-se a idade, estrato, árvore e secção a que pertencia o disco.

Os discos foram embalados separadamente por árvore, em sacos de ráfia, e encaminhados até a Embrapa Florestas, para em seguida medirem-se seus diâmetros e prepararem-se as amostras para determinações químicas e de densidade dos componentes casca e lenho.

A densidade básica do lenho e casca em cada secção do tronco foi determinada pelo método do máximo teor de umidade, devido à facilidade de execução em larga escala, pela dificuldade de imersão da casca e por não haver resultados significativamente diferentes entre esse método e o da balança hidrostática, conforme Foelkel; Brasil; Barrichello (1971). As amostras utilizadas para determinação dos nutrientes do componente lenho e casca foram secadas em estufas de ventilação forçada (temperatura $=$ $62,5 \pm 2,5^{\circ} \mathrm{C}$ ), até estabilização completa do seu peso, sendo, em seguida, trituradas em moinho tipo Wiley, passadas em peneira de malha 20 e armazenadas em vidros de utilização convencional para análises químicas.

Para a determinação dos teores de fósforo $(\mathrm{P})$, potássio $(\mathrm{K})$, cálcio $(\mathrm{Ca})$ e magnésio $(\mathrm{Mg})$, as amostras foram submetidas à digestão nitroperclórica e os teores determinados de acordo com a metodologia proposta por Sarruge e Haag (1974). O fósforo foi determinado pelo método Vanadomolibidato de amônia, o potássio por fotometria de chama, o cálcio e magnésio por absorção atômica. $\mathrm{O}$ nitrogênio foi obtido por digestão sulfúrica, seguida de determinação pelo método Micro Kjeldahl.

O volume total das árvores foi determinado pelo somatório do volume de cada segmento utilizando-se o método de Smalian. A massa/quantidade da matéria seca foi obtida multiplicando-se o

\footnotetext{
${ }^{1}$ Informação recebida por e-mail da engenheira Fabiana Maia (2005), ex-funcionária da Manasa S.A.
} 
volume de cada segmento pela respectiva densidade. Os conteúdos de nutrientes da casca e lenho foram calculados a partir da média da concentração dos nutrientes determinados nas extremidades de cada segmento (diâmetro maior e menor de cada segmento) e multiplicados pela massa seca correspondente ao segmento, obtendo-os pelo somatório dos conteúdos de cada segmento, da base à altura comercial da árvore.

Os dados de concentração e do seu respectivo conteúdo de nutrientes foram analisados pela utilização do sistema estatístico SAS, licenciado para Embrapa - Empresa Brasileira de Pesquisas Agropecuárias. Testaram-se os modelos lineares, seguidos pelos multiplicativos e exponenciais. No caso de regressão linear múltipla, efetuou-se a análise pelo procedimento Stepwise. A normalidade dos erros foi verificada pelo teste de Shapiro-Wilks, e a autocorrelação de primeira ordem, pela estatística de Durbin-Watson.

\section{RESULTADOS E DISCUSSÃO}

De posse dos dados do conteúdo dos nutrientes, foram obtidas as equações para estimativa dos nutrientes exportados pelo lenho.

Dentre os diversos modelos matemáticos, a seleção foi com base nos coeficientes de determinação $\left(\mathrm{R}^{2}\right)$, erro de estimativa do modelo, distribuição gráfica dos resíduos, ou seja, maior normalidade dos resíduos, em relação às respectivas equações (GUIMARÃES, 1985), valores da estatística de teste $\mathrm{F}$ e facilidade na obtenção das amostras ao longo do tronco (limitando-se à altura máxima de 4,0 m em relação ao nível do solo), como medida de praticidade na operacionalização da coleta do material.

Os modelos matemáticos selecionados foram submetidos à análise gráfica para os valores preditos dos nutrientes e à distribuição da normalidade dos resíduos pelo teste de Shapiro-Wilks (ROYSTON, 1992).

Ajustaram-se modelos lineares aos dados para cada variável dos nutrientes: nitrogênio, fósforo, potássio, cálcio e magnésio. A tabela 1 mostra que os valores de $\mathrm{R}^{2}$ são todos próximos de 1 , indicando como muito bom cada um dos ajustes. Os valores-p correspondentes ao teste F (na Anova) são todos próximos de zero, indicando que as hipóteses de parâmetros da análise de regressão são recusadas. Já os valores-p do teste de Shapiro-Wilks são todos menores que $5 \%$, logo, aceita-se a hipótese de normalidade para os resíduos.

Na tabela 1, são apresentados os modelos matemáticos que estimam o conteúdo dos nutrientes no lenho de $P$. taeda. Observa-se que os coeficientes de determinação $\left(\mathrm{R}^{2}\right)$, para os modelos que estimam os nutrientes, ficaram compreendidos entre 0,9564 e 0,9996 , e o maior valor do erro da estimativa foi de $13,15 \%$ para o modelo que estimou o potássio, enquanto que para o elemento fósforo foi de 7,93\%.

Observa-se que, para a estimativa do conteúdo total de nitrogênio, o modelo matemático utilizou como variável independente os conteúdos de nitrogênio, potássio, cálcio e magnésio, em dois segmentos, e o fósforo, em apenas um segmento. A maior simplicidade nos modelos utilizados para a determinação dos elementos fósforo, potássio e magnésio deve estar relacionada com o grau de importância e necessidade desses nutrientes para o crescimento do $P$. taeda, por sua maior homogeneidade quantitativa nas diferentes posições das árvores e independência com relação aos demais nutrientes.

A variável independente utilizada na estimativa do conteúdo total do fósforo foi o próprio conteúdo de $\mathrm{P}$, no segmento a 1,3 m e 3,0 m. Para o potássio, utilizou-se o conteúdo de $\mathrm{K}$ a 3,0 $\mathrm{m}$ e o Ca total. As variáveis independentes utilizadas para a determinação do conteúdo de cálcio total no lenho foram o $\mathrm{Ca}$ e $\mathrm{Mg}$ na base e a 2,0 m e Mg total, e, para a estimativa do conteúdo de magnésio total, utilizou-se o $\mathrm{Mg}$ a 3,0 m. Nota-se que os coeficientes de determinação que estimaram os conteúdos de nutrientes no lenho apresentaram valores entre 0,9667 e 0,9996, dando boa confiabilidade aos modelos. 
Tabela 1. Equações para determinação indireta dos nutrientes (g) acumulados no lenho de Pinus taeda, coeficientes de determinação $\left(\mathrm{R}^{2}\right)$, p-valor, erro da estimativa (Sxy \%), valores de $\mathrm{p}$-valor $<\mathrm{W}$, Durbin-Watson (D) e autocorrelação de $1^{\mathrm{a}}$ ordem do teste D da Anova da regressão.

Table 1. Equations to indirectly determine the nutrients (g) accumulated in the Pinus taeda wood, determination coefficients $\left(\mathrm{R}^{2}\right)$, $\mathrm{p}$-value, standard error of estimate in percent (Sxy \%), pvalue $<\mathrm{W}$ values, Durbin-Watson (D) and $1^{\text {st }}$ order autocorrelation of the regression Anova D test.

\begin{tabular}{|c|c|c|c|c|c|c|c|}
\hline Nutrientes & Equações & $\mathbf{R}^{2}$ & p-valor & Sxy $(\%)$ & p-valor $<W$ & D & ${ }^{\mathbf{r}} \mathbf{D}$ \\
\hline Nitrogênio & $\begin{array}{c}\mathrm{NT}=6,3720 \times \mathrm{N} 5+0,0238 \times \\
(\mathrm{N} \text { base })^{2}-11,9336 \times \mathrm{K} 3+6,4713 \times \\
\mathrm{Ca} 4-6,8791 \times \mathrm{Mg} \text { base }-3,3301 \times \\
(\mathrm{P} 3)^{2}+3,9417 \times(\mathrm{K} 2)^{2}-0,5030 \times \\
(\mathrm{Ca} 3)^{2}+3,9465 \times \mathrm{Mg} \mathrm{T}\end{array}$ & 0,9996 & $<0,0001$ & 10,03 & 0,9460 & 2,109 & $-0,058$ \\
\hline Fósforo & $\mathrm{PT}=7,0845 \times \mathrm{P} 3+6,6692 \times \mathrm{P} 5$ & 0,9667 & $<0,0001$ & 7,93 & 0,1648 & 1,237 & 0,353 \\
\hline Potássio & $\begin{array}{c}\mathrm{K} \mathrm{T}=-8,5675+9,1579 \times \mathrm{K} 5+ \\
0,2641 \times \mathrm{Ca} \mathrm{T}\end{array}$ & 0.9736 & $<0,0001$ & 13,15 & 0,3023 & 2,160 & $-0,091$ \\
\hline Cálcio & $\begin{array}{c}\mathrm{CaT}=1,6387+7,5308 \times \mathrm{Ca} 4- \\
28,1487 \times \mathrm{Mg} 4+0,0403 \times \\
(\mathrm{Ca} \text { base })^{2}-0,1815 \times(\mathrm{Mg} \text { base })^{2}+ \\
3,2009 \times \mathrm{Mg} \mathrm{T}\end{array}$ & 0,9953 & $<0,0001$ & 9,47 & 0,3076 & 1,524 & 0,205 \\
\hline Magnésio & $\mathrm{MgT}=14,34179 \times \mathrm{Mg} 5$ & 0,9730 & $<0,0001$ & 7,94 & 0,1855 & 1,263 & 0,294 \\
\hline
\end{tabular}

$\mathrm{NT}=\mathrm{N}$ Total $\mathrm{N} 1=\mathrm{N}$ base $-1,0 \mathrm{~m} ; \mathrm{N} 2=\mathrm{N} 1,0 \mathrm{~m}-1,3 \mathrm{~m} ; \mathrm{N} 3=\mathrm{N} 1,3 \mathrm{~m}-2,0 \mathrm{~m} ; \mathrm{N} 4=\mathrm{N} 2,0 \mathrm{~m}-3,0 \mathrm{~m} ; \mathrm{N} 5=\mathrm{N} 3,0-4,0 \mathrm{~m}$. Idem para $\mathrm{P}, \mathrm{K}, \mathrm{Ca}$ e $\mathrm{Mg}$.

W: teste de Shapiro-Wilks para normalidade dos resíduos.

D: Durbin-Watson; ${ }^{\mathrm{r}} \mathrm{D}$ : autocorrelação de $1^{\mathrm{a}}$ ordem do teste D.

Os resultados apresentados na tabela 2 foram obtidos com a utilização das equações apresentadas na tabela 1, inserindo-se os valores das variáveis independentes. Esses valores foram calculados por classes de diâmetro, devido à alta relação entre o diâmetro e o conteúdo de nutriente.

Tabela 2. Conteúdo de nutrientes no lenho (g/árvore) por classe de diâmetro, calculado em função das equações selecionadas, para Pinus taeda.

Table 2. Contents of nutrients in the wood (g/tree) per diameter class, calculated through the selected equations, for Pinus taeda.

\begin{tabular}{lrrrrr}
\hline Classe de diâmetro & $\mathbf{N}$ & $\mathbf{P}$ & $\mathbf{K}$ & $\mathbf{C a}$ & $\mathbf{M g}$ \\
\hline $10,0-15,0$ & 66,36 & 2,68 & 31,38 & 26,37 & 10,22 \\
$20,0-24,9$ & 175,67 & 12,53 & 50,39 & 68,76 & 26,15 \\
$25,0-29,9$ & 276,86 & 31,34 & 90,23 & 144,59 & 47,51 \\
$30,0-34,9$ & 403,01 & 38,21 & 104,88 & 167,34 & 62,76 \\
$35,0-40,0$ & 542,56 & 35,32 & 186,39 & 322,91 & 118,63 \\
\hline Média & 292,89 & 24,02 & 92,66 & 145,99 & 53,05 \\
\hline
\end{tabular}

Com as equações selecionadas e dados apresentados na tabela 2, é possível a obtenção da estimativa dos nutrientes exportados, dependendo apenas das classes de diâmetro em que se encontre a floresta e do número de árvores por hectare no caso de um corte raso ou das árvores que serão retiradas pelos desbastes.

Na tabela 3 são apresentadas as equações selecionadas, tendo-se levado em consideração para a seleção, além do coeficiente de correlação $\left(\mathrm{R}^{2}\right)$, o erro da estimativa, a homogeneidade e distribuição dos resíduos e valor-p da análise de variância, significativo ao nível de $1 \%$ de probabilidade.

Da mesma forma que no lenho, as equações selecionadas para estimativa dos nutrientes na casca, no caso do nutriente nitrogênio, mostraram-se ser mais complexas que as dos demais nutrientes, entretanto mais simples que a similar equação para determinação do nitrogênio no lenho. Essa evidência pode estar associada à maior homogeneidade dos nutrientes ao longo das árvores e à maior estabilidade dos nutrientes na casca, por ser constituída principalmente por tecidos mortos, portanto, com menor intensidade da ciclagem bioquímica. 
Observa-se, na tabela 3, que para todos os nutrientes da casca, os coeficientes de correlação $\left(\mathrm{R}^{2}\right)$ foram elevados, variando de 0,9614 para o fósforo a 0,9925 para o nitrogênio, o que confere uma alta confiabilidade aos modelos. $\mathrm{O}$ erro da estimativa para todos os nutrientes também corrobora essa constatação, apresentando valores baixos, sendo 12,16 o maior erro para o elemento nitrogênio. Outra constatação da confiabilidade dos modelos está demonstrada nos valores-p do teste de Shapiro-Wilks, que foram menores que $5 \%$, logo, aceita-se a hipótese de normalidade para os resíduos.

Tabela 3. Equações para determinação indireta dos nutrientes (g) acumulados na casca de Pinus taeda, coeficientes de determinação $\left(\mathrm{R}^{2}\right)$, p-valor, erro da estimativa (Sxy \%), valores de $\mathrm{p}$-valor $<\mathrm{W}$, Durbin-Watson (D) e autocorrelação de $1^{\text {a }}$ ordem do teste D da Anova da regressão.

Table 3. Equations to indirectly determine the nutrients ( $\mathrm{g}$ ) accumulated in the bark of Pinus taeda, determination coefficients $\left(\mathrm{R}^{2}\right)$, p-value, standard error of estimate (Sxy \%), p-value $<\mathrm{W}$ values, Durbin-Watson (D) and $1^{\text {st }}$ order autocorrelation of the regression Anova D test.

\begin{tabular}{|c|c|c|c|c|c|c|c|}
\hline Nutrientes & Equações & $\mathbf{R}^{2}$ & p-valor & Sxy (\%) & p-valor $<\mathbf{W}$ & D & ${ }^{\mathrm{r}} \mathrm{D}$ \\
\hline Nitrogênio & $\begin{array}{l}\mathrm{N}-\mathrm{T}=6,9187 \times \mathrm{N} 5+0,0566 \\
\mathrm{x}(\mathrm{N} \text { base })^{2}+25,2277 \times \mathrm{Mg} 4\end{array}$ & 0,9925 & $<0,0001$ & 12,16 & 0,4476 & 2,075 & $-0,040$ \\
\hline Fósforo & $\mathrm{P}_{-} \mathrm{T}=11,1199 \times \mathrm{P} 4$ & 0,9614 & $<0,0001$ & $1,5 \mathrm{E}-06$ & 0,6570 & 2,237 & $-0,164$ \\
\hline Potássio & $\begin{array}{c}\mathrm{K} \_\mathrm{T}=11,6991 \times \mathrm{K} 2+ \\
7,4758 \times \mathrm{K} 5\end{array}$ & 0,9765 & $<0,0001$ & 1,94 & 0,4278 & 1,410 & 0,292 \\
\hline Cálcio & $\begin{array}{l}\mathrm{Ca} \_\mathrm{T}=5,0936 \times \mathrm{Ca} 2+ \\
9,0312 \times \mathrm{Ca} 5\end{array}$ & 0,9888 & $<0,0001$ & 6,43 & 0,2471 & 1,197 & 0,396 \\
\hline Magnésio & $\mathrm{Mg} \_\mathrm{T}=12,3608 \times \mathrm{Mg} 5$ & 0,9849 & $<0,0001$ & 0,23 & 0,8850 & 1,158 & 0,396 \\
\hline
\end{tabular}

$\mathrm{NT}=\mathrm{N}$ Total; $\mathrm{N} 1=\mathrm{N}$ base $-1,0 \mathrm{~m} ; \mathrm{N} 2=\mathrm{N} 1,0 \mathrm{~m}-1,3 \mathrm{~m} ; \mathrm{N} 3=\mathrm{N} 1,3 \mathrm{~m}-2,0 \mathrm{~m} ; \mathrm{N} 4=\mathrm{N} 2,0 \mathrm{~m}-3,0 \mathrm{~m} ; \mathrm{N} 5=\mathrm{N} \mathrm{3,0-4,0} \mathrm{m}$.

Idem para $\mathrm{P}, \mathrm{K}, \mathrm{Ca}$ e $\mathrm{Mg}$.

W: teste de Shapiro-Wilks para normalidade dos resíduos; D: Durbin-Watson; ${ }^{\mathrm{r}}$ D: autocorrelação de $1^{\mathrm{a}}$ ordem do teste D.

Nota-se na tabela 3 que para todos os nutrientes as equações selecionadas são simplificadas em relação àquelas obtidas no lenho. No caso do nutriente fósforo e magnésio, seus próprios teores em uma única posição da árvore determinam os valores totais: para o fósforo na posição de 3,0 $\mathrm{m}$ e para o magnésio a determinação é definida pelo teor na altura de 4,0 $\mathrm{m}$ da árvore, possibilitando uma alta confiabilidade na estimativa.

Para a determinação do nitrogênio, único nutriente na casca cujo polinômio é constituído por dois elementos, são necessárias as determinações das variáveis independentes do próprio nutriente na base e a 4,0 m, o magnésio a 3,0 m, e, para o potássio e cálcio, pelos seus próprios teores nas alturas de $1,0 \mathrm{~m} \mathrm{e} 4,0 \mathrm{~m}$.

$\mathrm{Na}$ tabela 4 são apresentados os conteúdos de nutrientes estimados através das equações selecionadas na tabela 3 e em função dos conteúdos por classe dos diâmetros encontrados nas análises químicas determinadas nas árvores coletadas.

Tabela 4. Conteúdo de nutrientes na casca (g/árvore) por classe de diâmetro calculado em função das equações selecionadas, para Pinus taeda.

Table 4. Contents of nutrients in the bark (g/tree) per diameter class, calculated through the selected equations, for Pinus taeda.

\begin{tabular}{lccccc}
\hline Classe de diâmetro & $\mathbf{N}$ & $\mathbf{P}$ & $\mathbf{K}$ & $\mathbf{C a}$ & $\mathbf{M g}$ \\
\hline $10,0-15,0$ & 14,63 & 0,65 & 5,07 & 5,39 & 1,69 \\
$20,0-24,9$ & 34,08 & 2,27 & 10,76 & 16,57 & 3,10 \\
$25,0-29,9$ & 63,37 & 3,16 & 15,14 & 31,08 & 6,04 \\
$30,0-34,9$ & 93,67 & 4,34 & 23,22 & 59,71 & 9,44 \\
$35,0-40,0$ & 183,16 & 9,08 & 25,53 & 107,85 & 17,67 \\
\hline Média & 77,78 & 3,90 & 15,94 & 44,12 & 7,59 \\
\hline
\end{tabular}

Na tabela 4 são encontrados os conteúdos de nutrientes obtidos através das equações selecionadas que possibilitam a estimativa dos nutrientes exportados através dos desbastes ou corte final da floresta, bastando apenas o conhecimento do número de árvores por hectare e sua classe de diâmetro. 


\section{CONCLUSÕES}

Os resultados obtidos indicam as seguintes conclusões:

- A amostragem aleatória em povoamentos de P. taeda em diferentes idades, estratos vegetativos das árvores (dominante, co-dominante e dominada), diferentes condições de manejo e diferentes produtividade, permite obter equações de estimativa de acúmulo de nutrientes com altos índices de correlações e confiabilidade.

- A obtenção de amostragem em segmentos até 4,0 $\mathrm{m}$ de altura possibilita determinar o conteúdo total dos nutrientes nas árvores, sem necessidade de amostragens nos segmentos de posição mais elevada em relação ao topo da árvore.

- A determinação do nutriente nitrogênio apresenta um maior grau de complexidade e requer basicamente que outros macronutrientes também sejam determinados, o que demanda maiores custos amostrais e laboratoriais.

\section{AGRADECIMENTOS}

À Universidade Federal do Paraná, pela contribuição no aprimoramento científico, o qual possibilitou a realização deste trabalho. Às empresas Manasa-Madereira Nacional S.A. e Santa Maria Papel e Celulose, pela cessão do material florestal e auxílio pessoal nas coletas das amostras em campo. À Embrapa - Empresa Brasileira de Pesquisas Agropecuárias, pelo apoio nas análises laboratoriais e cessão das instalações para preparação das amostras.

\section{REFERÊNCIAS}

ANDRAE, F.; KRAPFENBAUER, A. Inventário de um reflorestamento de Araucária de 17 anos em Passo Fundo, RS. Parte II: Inventário de nutrientes. In: Pesquisas áustro-brasileiras 1973 - 1982 sobre Araucária angustifólia, Podocarpus lambertti e Eucalyptus saligna. Santa Maria: Universidade de Santa Maria, 1983. p. 30-55.

BURKHART, H. E. Data collection and modeling approaches for forest growth and yield prediction. In: Predicting Forest Growth and Yield: Current issues, future prospects. Seatle: University Washington, 1987. p. 3-16.

DANIELS, R. F.; BURKHART, H. E.; SPITLE, G. D.; SOMERS, G. L. Methods for modeling individual tree groth and stand development in seeded Loblolly pine stands. Blacksburg: Virginia Polytechnic Inst. and State Univ, 1979. (Publication FWS-1-79)

EMBRAPA. Carta de levantamento de reconhecimento dos solos do Paraná. Brasília, 1984.

FOELKEL, C. E. B.; BRASIL, M. A. M.; BARRICHELlO, L. E. G. Métodos para determinação da densidade básica de cavacos para coníferas e folhosas. IPEF, Piracicaba, n. 2/3, p. 65-74, 1971.

GUIMARÃES, D. F. Estimativa do volume de Eucalyptus spp através de posições absolutas no tronco. 96 f. Dissertação (Mestrado em Engenharia Florestal) - Universidade Federal do Paraná, Setor de Ciências Florestais. Curitiba, 1985.

REIS, M. G. F.; KIMMINS, J. P.; REZENDE, G. C.; BARROS, N. F. Acúmulo de biomassa em uma seqüência de idade de Eucalyptus grandis plantado no cerrado em duas áreas com diferentes produtividades. Revista Árvore, Viçosa, MG, v. 9, n. 2, p.149-162, 1985.

ROYSTON, P. Approximating the Shapiro-Wilk W-Test for normality. Statistics and Computing, London, n. 2, p. 117-119, 1992.

SARRUGE, J. R.; HAAG, H. P. Análise química em plantas. Piracicaba: ESALQ/USP. 1974. 56 p.

SCOLFORO, J. R. S. O sistema PISAPRO. Lavras, MG: UFLA/FAEPE. 1976b. 99 p. 
SCOLFORO, J. R. S.; MACHADO, S. A. Curvas de Índice de sítio para plantações de Pinus elliottii nos estados do Paraná e Santa Catarina. Revista Floresta, Curitiba, v. 18, n. 1/2, p. 140-158, 1988.

SOCIEDADE BRASILEIRA DE SILVICULTURA. O setor florestal brasileiro: fatos e números. São Paulo, 1998. $18 \mathrm{p}$.

SILVA, H. D. da. Modelos matemáticos para a estimativa da biomassa e do conteúdo de nutrientes em plantações de Eucalyptus grandis Hill (ex-Maiden) em diferentes idades. $101 \mathrm{f}$. Tese (Doutorado em Engenharia Florestal) - Setor de Ciências Agrárias, Universidade Federal do Paraná, Curitiba, 1996.

UNITE STATE. Department of Agriculture. Forest Service. Technology transfer, Pinus taeda. Disponível em: <http:// www2.fpl.fs.fed.us/techSeets/SoftwoodNA/htmlDocs/pinustaeda.html $>$. Acesso em: 16/10/2005.

VALERI, S. V. Exportação de biomassa e nutrientes de povoamentos de Pinus taeda L. desbastados em diferentes idades. $164 \mathrm{f}$. Tese (Doutorado em Engenharia Florestal) - Setor de Ciências Agrárias, Universidade Federal do Paraná, Curitiba, 1988.

VALOR FLORESTAL. Mercado de toras. In: CONGRESSO INTERNACIONAL DO PINUS, 2004, Joinville. [Anais...]. [S.1.: s.n.], 2004.

VAN LEAR, D. H., WAIDE, J. B.; TEUKE, M. J. Biomass and nutrient content of a 41-years-old loblolly pine (Pinus taeda L.) plantation on a poor site in South Carolina. Forest Science, Lawrence, v. 30, n. 2, p. 395-404, 1984. 